\title{
Submucosal pocket creation using a traction device in colorectal endoscopic submucosal dissection
}

\section{Daisuke Ide ${ }^{\mathrm{a}, \mathrm{b}}$, Shoichi Saito ${ }^{\mathrm{a}}$, Akiko Chino ${ }^{\mathrm{a}}$, Tomohiko Richard Ohya ${ }^{\mathrm{c}}$}

The Cancer Institute Hospital of Japanese Foundation for Cancer Research; The Jikei University School of Medicine, Tokyo, Japan

Colorectal endoscopic submucosal dissection (ESD) can be difficult for various reasons, and a thin muscular layer is associated with increased perforation rates and longer procedure times. The submucosal pocket creation method was recently reported to overcome these difficulties $[1,2]$. Mucosal flap formation is an important and difficult initial step. In contrast, several countertraction systems that facilitate ESD procedures for colorectal cancers have been reported [3]. We combined the submucosal pocket creation method with a traction device (TD) to achieve a safer and more efficient colorectal ESD procedure. The TD was designed to be fixed with nylon thread and clips on the free edges of mucosal overlays to deflect the diseased mucosa away from the dissection plane (Fig. 1). The key feature is the connection of the TD to the anal mucosa at approximately $10 \mathrm{~mm}$ from the distal side of the tumor, after submucosal injection prior to the initial mucosal incision (Fig. 2A). It is possible to attach the TD with adequate submucosal injection and to prevent complications, such as clip attachment to the muscle layer, as incision and dissection have not been performed (Fig. 2B). When repeated minimal incision and dissection were performed in this case, the mucosa was grasped with the TD to allow for exposure of the submucosa (Fig. 2C). Mucosal flap formation and submucosal pocket creation using the TD became easier (Fig. 2D). The submucosal cutting layer was clearly visualized and the appropriate amount of tension was created using the TD and submucosal pocket, facilitating dissection.

Department of aLower GI Medicine, The Cancer Institute Hospital of Japanese Foundation for Cancer Research (Daisuke Ide, Shoichi Saito, Akiko Chino); 'Internal Medicine, Division of Gastroenterology and Hepatology (Daisuke Ide); ${ }^{c}$ Endoscopy (Tomohiko Richard Ohya), The Jikei University School of Medicine, Tokyo, Japan

Conflict of Interest: None

Correspondence to: Daisuke Ide, MD, PhD, Department of Lower GI Medicine, The Cancer Institute Hospital of Japanese Foundation for Cancer Research, 3-8-31, Ariake, Koto, Tokyo 135-8550, Japan, e-mail: dei0405@gmail.com

Received 17 February 2018; accepted 27 February 2018 Published online 18 April 2018

DOI: https://doi.org/10.20524/aog.2018.0258

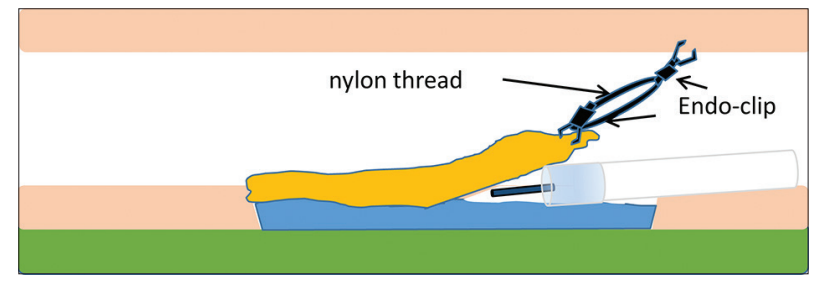

Figure 1 Diagram of nylon thread traction device
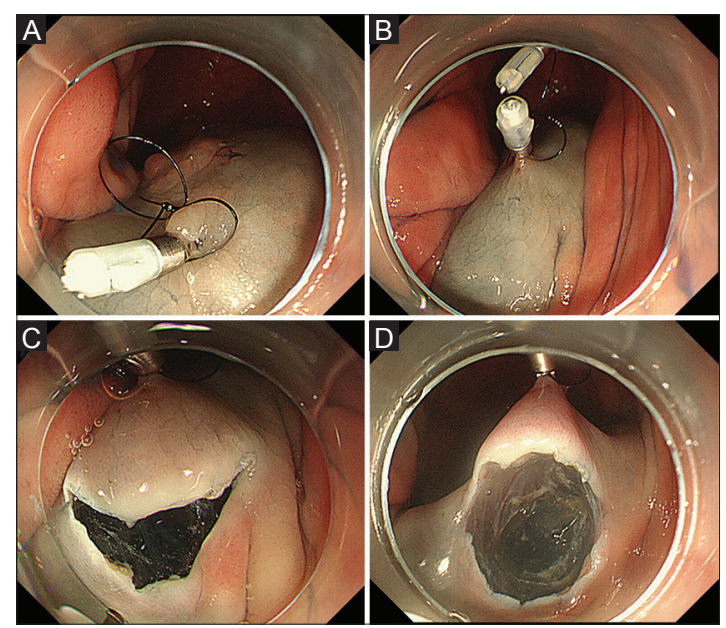

Figure 2 Procedure of submucosal pocket creation with a traction device for colorectal endoscopic submucosal dissection. (A, B) The traction device is connected to the anal mucosa from the distal side of the tumor after submucosal injection, without an initial mucosal incision. (C) A minimal incision is made from the distal side of the connected traction device. (D) Submucosal pocket creation is facilitated when repeated minimal incision and dissection are performed

\section{References}

1. Hayashi Y, Sunada K, Takahashi H, et al. Pocket-creation method of endoscopic submucosal dissection to achieve en bloc resection of giant colorectal subpedunculated neoplastic lesions. Endoscopy 2014;46(Suppl 1 UCTN):E421-E422.

2. Sakamoto H,HayashiY,Miura Y, etal. Pocket-creation method facilitates endoscopic submucosal dissection of colorectal laterally spreading tumors, non-granular type. Endosc Int Open 2017;5:E123-E129.

3. Sakamoto N, Osada T, Shibuya T, et al. Endoscopic submucosal dissection of large colorectal tumors by using a novel spring-action S-O clip for traction (with video). Gastrointest Endosc 2009;69:1370-1374. 